\title{
DIAMOND GENESIS REVEALED BY X-RAY TOMOGRAPHY OF DIAMONDIFEROUS ECLOGITES
}

\author{
Mahesh Anand* ${ }^{1}$, Lawrence A. Taylor ${ }^{1}$, William D. Carlson ${ }^{2}$, Dawn-Hwa Taylor ${ }^{1}$ and Nikolai V. \\ Sobolev $^{3}$ \\ ${ }^{I}$ Planetary Geosciences Institute, Department of Geological Sciences, University of Tennessee, Knoxville, TN 37996, USA \\ ${ }^{2}$ University of Texas, Austin, USA \\ ${ }^{3}$ Institute of Mineralogy \& Petrography, Russian Academy of Sciences, Novosibirsk 630090, Russia
}

\section{INTRODUCTION}

Significant advances in our understanding of mantle processes have resulted from the petrological studies of diamonds and their mineral inclusions (DIs). However, the physics and chemistry of the growth of diamonds and their relationships to their host eclogites and its mineralogy are not well understood. High-resolution Computed X-ray tomography (HRCXT) of diamondiferous eclogite xenoliths can be used to study the inter-relationship of their constituent minerals in 3dimensional space. Importantly, it is a non-destructive technique that maps and locates the diamonds and other minerals within xenoliths, thereby enabling one to investigate the nature of diamond formation in its original setting. As part of a comprehensive study of diamondiferous xenoliths, we have conducted HRCXT studies of several eclogites from the diamond mines in Yakutia, Siberia. This 3-D mapping was followed by extensive chemical and isotopic investigations of the host eclogite, DIs, and the diamonds themselves, some results of which have been presented elsewhere (Anand et al., this volume).

\section{HRCXT METHODOLOGY}

The principles of the HRCXT technique are similar to CAT-scans used in the medical profession. The term "CAT" stands for "computerized axial tomography". In this method, a highly sensitive X-ray beam is focused on a specific plane of the human body (e.g., the head). As this beam passes through the body, it is sensed by a detector, which feeds the information it receives into a computer. The computer then analyzes the information on the basis of tissue density. These analyzed data are then fed into a cathode-ray tube, and a picture of the Xrayed, cross-section of the body is produced. Bones and tissues appear as varying shades of gray, depending on their densities and X-ray absorption characteristics. These gray-scale images can be readily adjusted for any color scheme. The industrial X-ray tomographic analyzer used in this study is conceptually similar to a
CAT-scan, however, it is capable of significantly higher $\mathrm{X}$-ray intensity and markedly higher spatial resolution, on the order of microns (Carlson and Denison, 1992, Rowe et al., 1997). Rocks and fossils subjected to the high-resolution X-ray tomography can reveal the textural features of inside material in these objects, as well as the 3-D relationships among their various constituents.

\section{APPLICATION}

The rare specimens of diamondiferous eclogite xenoliths make them invaluable samples of the Earth's mantle in which diamonds originally formed. To better understand the mantle setting of the diamonds within their host rocks, a non-destructive method of study is preferred over the conventional methods used in most petrological studies. The HRCXT provides us a unique opportunity to investigate the spatial and textural relationships between diamonds and other minerals in the host eclogite, by this non-destructive method. Based on these mapping surveys, proper planning can be made for detailed dissection of the eclogite. This ensures maximum recovery of diamonds from an eclogite while ensuring minimum loss of material. In addition, it also allows us to preserve the original fabric

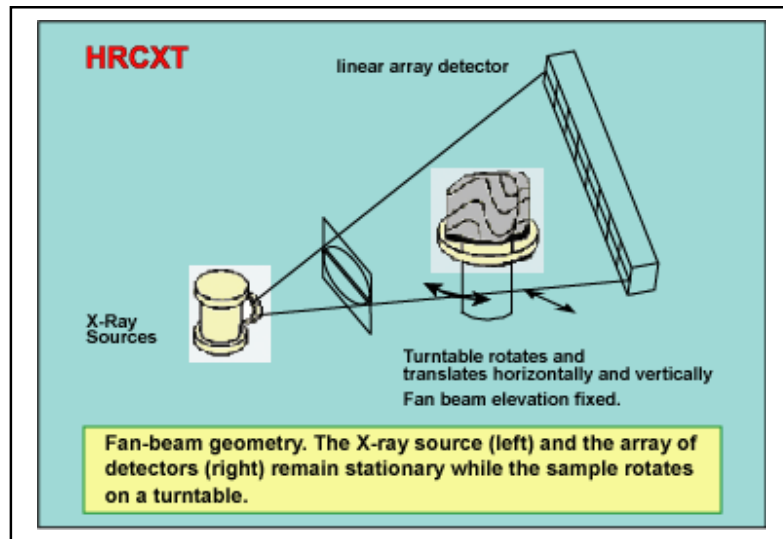

Fig. 1 Cartoon illustrating the principle involved in the HRCXT technique. 
and textural features of the eclogites, even after dissecting the sample into several hundreds of pieces.

The HRCXT images used in our studies were obtained at the University of Texas, Austin, as a series of 350400 2-D slices each, of a diamondiferous eclogite, using a micro-focal X-ray source and an image-intensifier detector system to measure the absorption of X-rays along numerous coplanar paths through the sample. The plane containing the X-ray paths is divided into a matrix of pixels thereby creating a 2-D image. A cartoon illustrating the basic methodology of the technique is shown in Figure 1. Different minerals have different X-ray attenuation values; thus, in the 2-D image, they appear as different shades of color. In this manner, it is easy to differentiate between diamonds, sulfides, silicates, and their alteration products in a xenolith. Several such 2-D images are stacked together using volume-visualization software to produce a 3-D model of the xenolith. This essentially represents a density map of the sample, from which one can extract the sizes, shapes, textures, and locations of individual crystals that have dimensions larger than the spatial resolution of the scans. Such 3-D models reveal clearly the spatial relationships between diamonds and their surroundings. This provides clues to the processes that control diamond crystallization. These relationships are determined by rotating and viewing the model at different perspectives to look for any mineral associations, alignments, or fabric. It is possible to make some of the phases transparent and display only selected mineral phases at a time. For example, a 3-D HRCXT model of diamondiferous eclogite, UX-1, from the Udachnaya kimberlite pipe, Yakutia, is shown in Figure 2. For clarity, the garnet and clinopyroxene grains are made invisible so that locations and sizes of diamonds can be easily seen. A total of 69 macrodiamonds are clearly visible in the interior of this sample, which otherwise would not have been seen by any other technique. Many of the diamonds in the eclogite seem to be linearly oriented along a vertical axis passing through the central region of the eclogite. This observation aided our positioning of cuts through this sample parallel to this axis such that maximum

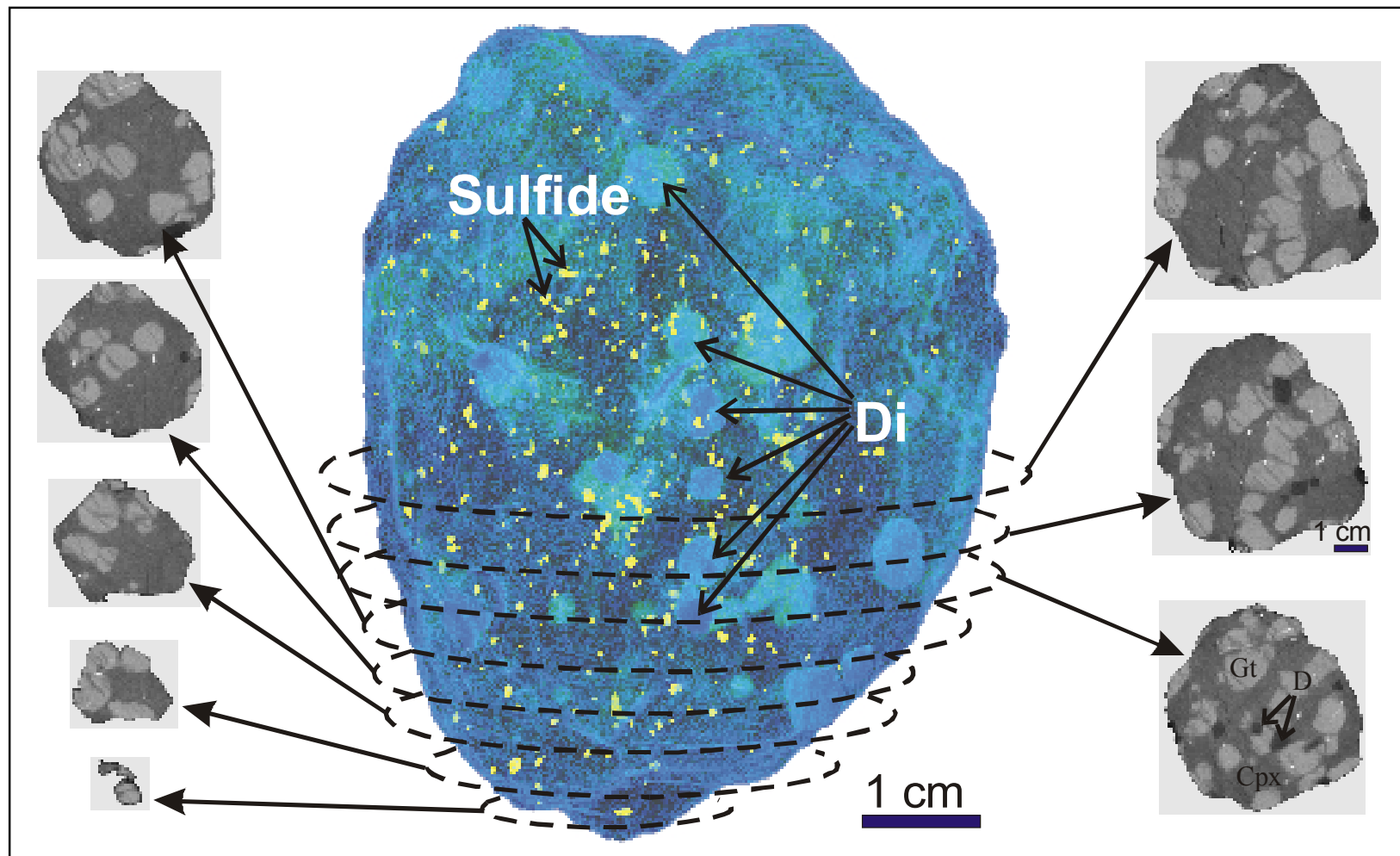

Fig. 2 Center- A 3-D model of diamondiferous eclogite xenolith, UX-1 based upon results of high-resolution computed $\mathrm{X}$-ray tomography. Blue grains are diamonds, and yellow specs are sulfide minerals distributed throughout the sample. Garnet and clinopyroxene crystals were rendered invisible to improve the clarity. 6 diamonds are seen aligned parallel to the central vertical axis. Figures on left and right of the central image are individual 2-D X-ray slices obtained originally by the HRCXT technique. The dotted girdle around the eclogite indicates the level from which each 2-D slice was obtained. In these slices, diamond appears black, garnet as light gray, Cpx as dark gray, and sulfide as white. 
number of diamonds could be exposed on the cut of a Quick Time movie at the following website: surface.

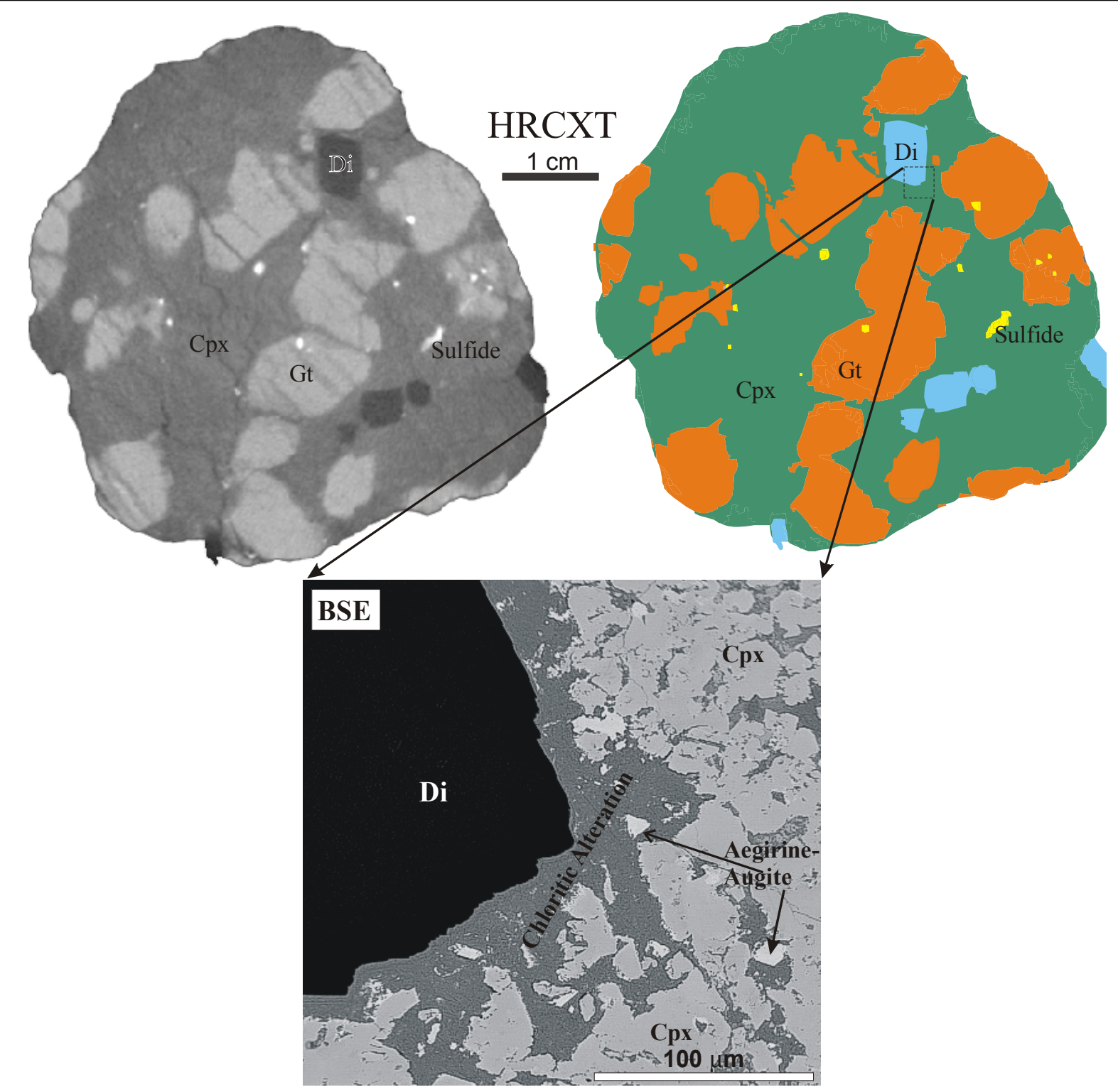

Fig. 3 Top - Grayscale and colored versions of a 2-D X-ray slice from the eclogite xenolith, UX-1. Bottom - BSE image of an area of a thin section, represented by the small rectangular box in the top right image. The thin-section was prepared such that it included the contact between the diamond and the host minerals, as seen in bottom figure. A zone of secondary alteration always found rimming the diamond in the eclogite host. Patches of aegirine augite, low$\mathrm{Na} \mathrm{Cpx}$, amphibole, and chlorite constitute these alteration zones.

In Figure 2, on either side of the central image, 8 of the total 360 individual 2-D slices are shown that were originally collected by the X-ray analyzer. Here, only every $20^{\text {th }} 2$-D slice has been shown. [A full sequence of all 360 slices of this xenolith can be seen in the form the 3-D model, as shown in Figure 2 can be seen on the same website, where viewers can spin the model 360 degrees, along a vertical axis to get the desired orientation.] In Figure 2, each 2-D X-ray slice is sequentially arranged from bottom to the middle part of the eclogite, corresponding to a particular level from 
which they were obtained. These sequential 2-D images show the appearance and disappearance of diamonds as one moves from bottom towards the top of the eclogite.

The in-plane spatial resolution of this technique is of the order of 10-20 $\mu \mathrm{m}$, which greatly aided the imaging of even the micro-diamonds $(<1 \mathrm{~mm})$. These diamonds in the Siberian eclogites (74 macro-diamonds in one $66 \mathrm{~g}$ eclogite $=144,000 \mathrm{cts} / \mathrm{ton})$ are all associated with alteration zones with prominent subplanar fabric of secondary mineralization. The alteration zones typically represent products of metasomatically induced partial melting (Spetsius and Taylor, 2002) and appear prominently on 2-D HRCXT images due to their lower density (Fig. 2, 3). It is interesting to note that diamond was never observed in direct contact or enclosed within fresh garnet or clinopyroxene. Furthermore, sulfide minerals are not preferentially associated with diamond. The association of diamonds with secondary minerals suggests that the diamonds formed after the eclogite, perhaps in conjunction with metasomatic input(s) of C$\mathrm{O}-\mathrm{H}-\mathrm{N}-\mathrm{S}$ fluids. It is also possible that the presence of diamonds enhanced the permeability contrast between the diamond and host-eclogite minerals that was subsequently used by pervading metasomatic fluids. Based on these observations, and our other studies on diamond inclusions and minerals in host eclogites (Anand et al., this volume; Taylor et al., 2000), it is positively concluded that the diamonds are not syngenetic with the garnet and clinopyroxene in the host eclogites.

\section{CONCLUDING REMARKS}

HRCXT is a powerful tool for mapping diamonds and other minerals in xenoliths. The Udachnaya eclogite xenoliths currently under investigation are exceptionally rich in diamonds: i.e., $>100,000$ carats/ton); $>70-90 \%$ of the total diamonds in these xenoliths occur in the interior of the xenolith and are only discovered by the use of HRCXT, without destroying the sample. Diamonds seem to be always near omphacite, along zones with a prominent subplanar fabric of secondary mineralization. Sulfide minerals, although abundant, are not preferentially associated with diamonds; there is insufficient sulfide mineralization for diamond formation from an immiscible sulfide melt. Diamonds in secondary minerals strongly implies metasomatic (C-O-H-N-S fluids) formation of diamonds, post-dating the formation of the host eclogite. These observations are also consistent with our other studies that suggest that diamonds are not syngenetic with the garnet and clinopyroxene in the host eclogite (Anand et al., this volume; Taylor et al., this volume; Taylor et al., 2000; Spetsius and Taylor, 2002).

\section{REFERENCES}

Anand, M., Taylor, L. A., Carlson, W. D., Taylor, D-H., Sobolev, N. V. 2003. Diamond genesis revealed by Xray tomography of diamondiferous eclogites. This volume.

Carlson, W. D., Denison, C. 1992. Mechanisms of porphyroblast crystallization: Results from HighResolution Computed X-ray Tomography. Science, 257, 1236-1239.

Misra, K. C., Anand, M., Taylor, L. A. 2003. Multistage alteration of (two?) diamondiferous eclogite xenoliths from the Udachnaya kimberlite pipe, Yakutia, Russia. Submitted (Contrib. Min. Pet.).

Rowe, T., Kappelman, J., Carlson, W. D., Ketcham, R. A., Denison, C. 1997. High-resolution computed tomography: A breakthrough technology for earth scientists. Geotimes, 23-27.

Spetsius, Z. V., Taylor, L. A. 2002. Partial melting in mantle eclogite xenoliths: Clues to microdiamond genesis. Int. Geol. Rev. 44, 973-987.

Taylor, L. A., Anand, M., Promprated, P. 2003. Diamonds and their inclusions: Are criteria for syngenesis valid? This volume.

Taylor, L. A., Keller, R. A., Snyder, G. A., Wang, W. Y., Carlson, W. D., Hauri, E. H., McCandless, T., Kim, K. R., Sobolev, N. V. 2000. Diamonds and their mineral inclusions, and what they tell us: A detailed "pullapart" of a diamondiferous eclogite. Int. Geol. Rev. 42, 959-983. 